\title{
Polymorphic variants of genes involved in choline pathway and the risk of intrauterine fetal death
}

\author{
Krzysztof Drews ${ }^{1}$, Agata Różycka ${ }^{2,}{ }^{3}$, Magdalena Barlik ${ }^{1}$, Andrzej Klejewski ${ }^{4,}$, \\ Grażyna Kurzawińska ${ }^{2}$, Hubert Wolskii, 6 , Marian Majchrzycki ${ }^{7}$, Agnieszka Gryszczyńska ${ }^{8}$, \\ Adam Kamiński ${ }^{9}$, Agnieszka Seremak-Mrozikiewicz ${ }^{1,2,8}$ \\ ${ }^{1}$ Division of Perinatology and Women's Diseases, Poznan University of Medical Sciences, Poznan, Poland \\ ${ }^{2}$ Laboratory of Molecular Biology in Division of Perinatology and Women's Diseases, Poznan University of Medical Sciences, \\ Poznan, Poland \\ ${ }^{3}$ Department of Biochemistry and Molecular Biology, Poznan University of Medical Sciences, Poznan, Poland \\ ${ }^{4}$ Department of Nursing, Poznan University of Medical Sciences, Poznan, Poland \\ ${ }^{5}$ Department of Obstetrics and Women's Diseases, Poznan University of Medical Sciences, Poznan, Poland \\ ${ }^{6}$ Division of Gynecology and Obstetrics, Podhale Multidisciplinary Hospital, Nowy Targ, Poland \\ ${ }^{7}$ Department of Rheumatology and Rehabilitation, Poznan University of Medical Sciences, Poland \\ ${ }^{8}$ Department of Pharmacology and Phytochemistry, Institute of Natural Fibers and Medicinal Plants, Poznan, Poland \\ ${ }^{9}$ Department of Pediatric Orthopaedics and Traumatology, Pomeranian Medical University, Szczecin, Poland
}

\begin{abstract}
Objectives: Choline and folate metabolism disturbances may be involved in the occurrence of intrauterine fetal death (IUFD). The proper activity of this metabolism could be determined by genetic variants involved in choline pathway e.g. CHKA (gene encoding choline kinase a), PCYT1A (gene encoding CCTa) and CHDH (gene encoding choline dehydrogenase). Our study aimed at determining the genotype and allele frequencies of CHKA rs7928739, PCYT1A rs712012, PCYT1A rs7639752, CHDH rs893363 and CHDH rs2289205 polymorphisms in mothers with IUFD occurrence.

Material and methods: The study involved 76 mothers with IUFD occurrence and 215 mothers of healthy children. Genetic analysis was performed with the use of PCR/RFLP method.

Results: The frequency of genotypes and alleles of studied polymorphisms was similar in both groups. The study revealed no association of PCYT1A, CHKA and CHDH polymorphisms in analysed groups of women. While evaluating the co-existence of analysed polymorphisms statistically significant correlation was revealed. Co-existence of $\mathrm{CHKA} \mathrm{rs7928739} \mathrm{AC/CHDH}$ rs2289205 AA genotypes was observed statistically more frequently in the study group than in the control group ( $p=0,031)$.

Conclusions: There is no correlation between single CHKA rs7928739, PCYT1A rs712012, PCYT1A rs7639752, CHDH rs893363 and $\mathrm{CHDH}$ rs2289205 polymorphisms and the incidence of intrauterine fetal death. However, revealed statistically significant difference between co-existence of CHKA rs7928739 AC/CHDH rs2289205 AA genotypes between study groups suggest the need of further analysis.
\end{abstract}

Key words: choline, intrauterine fetal death, genetic polymorphism

Ginekologia Polska 2017; 88, 4: 205-211

\section{INTRODUCTION}

Choline is a nutrient essential for proper fetal and placental development. Choline acts as a methyl group donor in the reaction of methylation of homocysteine into methionine. The reduced supply of choline-derived methyl donors may impair Hcy remethylation, leading to elevations in Hcy, and perturb folate mediated one-carbon metabolism (e.g., nucleotide biosynthesis and cellular methylation 
reactions) [1, 2]. Choline is used for production of phosphatidylcholine (PC) and sphingomyelin in the cycle called cytidine - diphosphocholine (CDP-choline) pathway. Phosphatidylcholine and sphingomyelin are essential for proper functioning of cell membranes, lipoprotein metabolism and moreover for the regulation of cellular life cycle $[3,4]$. PC is synthesized through the CDP-choline pathway and via the phosphatidylethanolamine $N$-methyltransferase (PEMT) de novo pathway. The CTP: phosphocholine cytidylyltransferase (CCT) catalyzes the key rate-limiting step in the CDP-choline pathway (Fig. 1) [3-5]. In human pregnancy, PEMT-phosphatidylcholine ( $v s$ phosphatidylcholine produced by the CDP-choline pathway) is preferentially partitioned from the maternal to the fetal compartment [6]. Thus, supplementing the maternal diet with extra choline during early pregnancy may increase supply of choline and DHA to the developing fetus.

$\mathrm{CHDH}$ gene encodes choline dehydrogenase localized in mitochondria. Variations of this gene may result in susceptibility to choline deficiency. Choline dehydrogenase is involved in the subpathway that synthesizes betaine aldehyde from choline (cytochrome c reductase route). Choline is imported through one of several transporters and phosphorylation by choline kinase (CHK) occurs in the cytoplasm (Fig. 1) [7]. There are two isoforms of choline kinase - $\mathrm{CHKa}$ and $\mathrm{CHK} \beta$, with the a as a dominant isoform [8]. The CHKA gene which encodes choline kinase $a$ is found at chromosome 11q13.2. CCT is the rate-controlling enzyme in the CDP-choline pathway and its activity is regulated

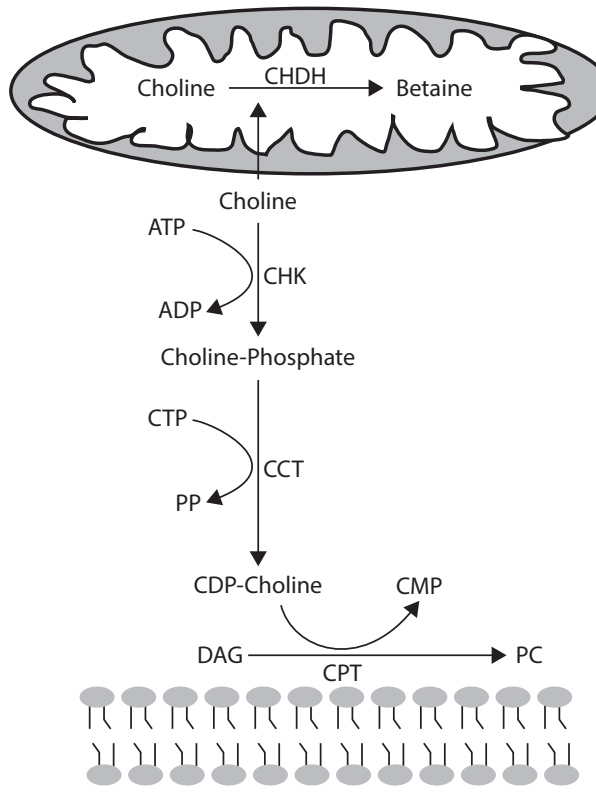

Figure 1. Choline pathways for phosphatidylcholine and betaine synthesis; PC — phosphatidylcholine; $\mathrm{CHDH}$ — choline dehydrogenase; CHK — choline kinase; CCT — CTP: phosphocholine cytidylyltransferase; DAG - diacylglycerol by signals from the membrane that report on the relative PC abundance. Human PCYT1A (chromosome 3q29) and PCYT1B (chromosome Xp22.11) encode CCTa and CCT $\beta$, respectively [9].

Given that pregnant women carrying genetic variants that increase choline requirements may be particularly susceptible to choline inadequacy during this reproductive state, we investigated the influence of CHKA, PCYT1A and $\mathrm{CHDH}$ genotypes on the risk of intrauterine fetal death (IUFD). Our study aimed at determining the incidence and genotype frequencies of CHKA rs7928739, PCYT1A rs712012, PCYT1A rs7639752, $\mathrm{CHDH}$ rs893363 and $\mathrm{CHDH}$ rs2289205 polymorphisms in 76 healthy mothers with IUFD of unknown origin occurrence (case mothers) and in 215 healthy mothers of healthy children (control mothers). Additionally, tests for association of $\mathrm{CHDH}, \mathrm{PCYT} 1 \mathrm{~A}$ and CHKA polymorphisms and evaluation of co-existence of analyzed genetic variants were performed.

\section{MATERIAL AND METHODS Study population}

Peripheral blood samples from 76 healthy mothers with IUFD of unknown origin were obtained from the Department of Perinatology and Women's Diseases, Poznan University of Medical Sciences. In addition, 215 mothers of healthy newborns born at term were used as controls. Patients with IUFD were enrolled to the study group within 2004-2014 year, and women to the control group - within 2012-2014 year. All women with improper fetal development, congenital malformations of the fetus, pregnancy connected diseases, placental and umbilical abnormalities, physical trauma during pregnancy, chronic diseases, vegan or vegetarian diet were excluded from the study as well as from the control group. Additionally, women with a positive history of IUFD in previous pregnancies were excluded from the control group. Moreover, each women involved in the analysis declared taking folic acid at least 3 months before conception and up to the end of pregnancy. Gestational age of IUFD was established on the basis of the date of the last menstruation, regularity of periods and ultrasound examinations. The clinical characteristic of the study and control groups is shown in Table 1. All participating women were Caucasian of Polish origin and were recruited from the same geographical region (Wielkopolska). The study protocols were approved by the local Ethics Committee. Written consent was obtained from all participants.

\section{Genotyping by RFLP}

Genotyping for polymorphisms in $\mathrm{CHDH}$ (rs893363 and rs2289205), PCYT1A (rs712012 and 7639752) and CHKA (rs7928739) was determined by polymerase chain reaction-restriction fragment length polymorphism (PCR-RFLP) 
assay. To amplify the gene fragments harboring these polymorphisms, five sets of the following primers were designed:

- CHDH rs893363 5'-GAGGAGCAACGTGGTGATCT-3'and 5' -CGAAGGAACCAGGAGGATAAG- 3'

- CHDH rs2289205 5'-CCACCTCAACCTCCAGTTGT-3' and 5' -GCAGCCATTCACTAACATGC- 3'

- PCYT1A rs712012 5'-GAGGGACAGAGGTCAAGGTG- 3' and $5^{\prime}$-AGTCACAAATCCCCTGAACG- 3'

- PCYT1A rs7639752 5' -CGCCCCTGTAGAGAACTGAC- 3' and 5'-ATATCCATAGCCCCAGGTGA- 3'

- CHKA rs7928739 5'-TGATTTCCAATGTCGAATCC- 3 ' and 5'-TAAGTCAAAATGCCGCTCTG- 3'

The analyzed polymorphic variants were identified by appropriate restriction enzyme digestion. The digested PCR products were analyzed on a $2 \%$ agarose gel and stained with ethidium bromide for visualization under UV light.

\section{Statistical analysis}

The genotype and allele frequencies of the analyzed polymorphisms were compared between both groups of mothers using a case-control study design. Significance was evaluated by the Fisher exact test. Odds ratios (ORs) and 95\% confidence intervals (Cls) were estimated using the GraphPad (Instant, USA) program. An online (http://ihg2.helmholtz-muenchen.de/cgi-bin/hw/hwa1.pl) program for deviation from the Hardy-Weinberg equilibrium was applied.

\section{RESULTS}

Table 2 presents genotype and allele distribution among mothers with IUFD and control mothers. As shown there were no statistically significant correlations between analyzed genetic variants and the incidence of IUFD. The frequency of genotypes and alleles of studied polymorphisms was similar in both groups. Additionally, tests for association of PCYT1 A, CHKA and CHDH polymorphisms in mothers with IUFD occurrence and control mothers was performed. The study revealed no association of single PCYT1A, CHKA and CHDH polymorphisms in analysed groups of women (Tab. 3).

While evaluating the co-existence of analysed polymorphisms statistically significant correlation was revealed. Co-existence of CHKA rs7928739 AC/CHDH rs2289205 AA genotypes was observed statistically more frequently in the study than in the control group $(p=0.031)$.

\section{DISCUSSION}

Intrauterine fetal death is a serious obstetrical complication. It is thought that globally there are at least 3.2 million stillbirths per year [10]. Infections, problems related to the placenta and umbilical cord, congenital anomalies, maternal

\begin{tabular}{|c|c|c|c|c|}
\hline & & $\begin{array}{l}\text { IUFD } \\
(n=76)\end{array}$ & $\begin{array}{l}\text { Control group } \\
\qquad(n=215)\end{array}$ & $\mathbf{p}$ \\
\hline Age (years) & $\begin{array}{l}\text { Mean } \pm \text { SD } \\
\text { Median } \\
\text { Min-max }\end{array}$ & $\begin{array}{c}30.46 \pm 4.32 \\
31 \\
20-42\end{array}$ & $\begin{array}{c}30.66 \pm 4.66 \\
31 \\
17-46\end{array}$ & 0.74 \\
\hline $\begin{array}{l}\text { Systolic pressure } \\
{[\mathrm{mm} \mathrm{Hg}]}\end{array}$ & $\begin{array}{l}\text { Mean } \pm \text { SD } \\
\text { Median } \\
\text { Min-max }\end{array}$ & $\begin{array}{c}105.80 \pm 11.46 \\
100.00 \\
90-140\end{array}$ & $\begin{array}{c}103.53 \pm 10.23 \\
100,00 \\
80-120\end{array}$ & 0.11 \\
\hline $\begin{array}{l}\text { Diastolic pressure } \\
{[\mathrm{mm} \mathrm{Hg}]}\end{array}$ & $\begin{array}{l}\text { Mean } \pm \text { SD } \\
\text { Median } \\
\text { Min-max }\end{array}$ & $\begin{array}{c}66.73 \pm 10.57 \\
60 \\
55-120\end{array}$ & $\begin{array}{c}65.79 \pm 7.99 \\
60 \\
50-90\end{array}$ & 0.42 \\
\hline Height [cm] & $\begin{array}{l}\text { Mean } \pm \text { SD } \\
\text { Median } \\
\text { Min-max }\end{array}$ & $\begin{array}{c}165.84 \pm 0.09 \\
167 \\
150-183\end{array}$ & $\begin{array}{c}166.84 \pm 5.71 \\
167 \\
151-184\end{array}$ & 0.30 \\
\hline Weight [kg] & $\begin{array}{l}\text { Mean } \pm \text { SD } \\
\text { Median } \\
\text { Min-max }\end{array}$ & $\begin{array}{c}62.01 \pm 9.92 \\
61 \\
44-99\end{array}$ & $\begin{array}{c}60.99 \pm 10.56 \\
59 \\
45-114\end{array}$ & 0.46 \\
\hline $\mathrm{BMI}\left[\mathrm{kg} / \mathrm{m}^{2}\right]$ & $\begin{array}{l}\text { Mean } \pm \text { SD } \\
\text { Median } \\
\text { Min-max }\end{array}$ & $\begin{array}{c}22.58 \pm 3.59 \\
21.61 \\
18.03-38.67\end{array}$ & $\begin{array}{c}21.89 \pm 3.58 \\
21.14 \\
16.94-40.53\end{array}$ & 0.15 \\
\hline IUFD & $\begin{array}{c}\text { One } \\
\text { Two or more } \\
\leq 37 \mathrm{gw} . \\
>37 \mathrm{gw} .\end{array}$ & $\begin{array}{c}69 \\
7 \\
72 \\
4\end{array}$ & $\begin{array}{l}0 \\
0 \\
0 \\
0\end{array}$ & - \\
\hline $\begin{array}{l}\text { Gestational week of } \\
\text { IUFD }\end{array}$ & $\begin{array}{l}\text { Mean } \pm \text { SD } \\
\text { Median } \\
\text { Min-max }\end{array}$ & $\begin{array}{c}30.02 \pm 4.92 \\
30 \\
22-40\end{array}$ & - & - \\
\hline
\end{tabular}


diseases and poor nutritional status have been reported to be the reasons of fetal death [11]. A number of investigations, including autopsy, examination of the placenta, amniocentesis and tests on blood from the mother and child, have been recommended in the diagnostic evaluation of IUFD [12-15]. The proportion of IUFDs in which no identifiable cause can be determined ranges from 12 to $50 \%$ and may be related to environmental factors [16-18]. Notably, increased consumption of dietary choline during pregnancy can improve biomarkers of choline metabolism. For example, consumption of 930 versus $480 \mathrm{mg}$ choline/day by third-trimester pregnant women led to higher circulating concentrations of several choline-derived methyl donors and restored choline partitioning between the CDP-choline and betaine pathways (which compete for choline as a substrate) to a nonpregnant state $[6,19]$.

Research on the role of dietary factors in human reproduction is limited but intake of certain nutrients, particularly folate, could positively influence reproductive success [20]. Numerous studies carried out on pregnant women have mentioned increased levels of homocysteine (Hcy) in relation to all the clinical conditions where a vascular-placental

Table 2. Genotype and allele distribution among mothers with intrauterine fetal death incidence (IUFD case mothers) and control mothers

\begin{tabular}{|c|c|c|c|c|c|c|c|}
\hline \multirow[t]{2}{*}{ Polymorphism } & \multirow[t]{2}{*}{$\begin{array}{l}\text { Group } \\
\text { of women }\end{array}$} & \multicolumn{5}{|c|}{$\begin{array}{c}\text { Genotype distribution absolute } \\
\text { number (frequency) }\end{array}$} & \multirow{2}{*}{$\begin{array}{l}\text { Allele P } \\
\text { Value }^{\text {a }} \\
\text { T vs. C }\end{array}$} \\
\hline & & TT & TC & CC & $\mathbf{T}$ & C & \\
\hline \multirow{2}{*}{$\begin{array}{l}\text { PCYT1A } \\
\text { rs712012 }\end{array}$} & Control mothers & $\begin{array}{c}78 \\
(0.36)\end{array}$ & $\begin{array}{l}107 \\
(0.50)\end{array}$ & $\begin{array}{c}30 \\
(0.14)\end{array}$ & $\begin{array}{c}263 \\
(0.61)\end{array}$ & $\begin{array}{l}167 \\
(0.39)\end{array}$ & $p=1.00$ \\
\hline & Case mothers & $\begin{array}{c}26 \\
(0.34)\end{array}$ & $41(0.54)$ & $\begin{array}{c}9 \\
(0.12)\end{array}$ & $\begin{array}{c}93 \\
(0.61)\end{array}$ & $\begin{array}{c}59 \\
(0.39)\end{array}$ & \\
\hline \multirow{3}{*}{$\begin{array}{l}\text { PCYT1A } \\
\text { rs7639752 }\end{array}$} & & AA & AG & GG & A & G & A vs. G \\
\hline & Control mothers & $\begin{array}{c}49 \\
(0.23)\end{array}$ & $\begin{array}{l}105 \\
(0.49)\end{array}$ & $\begin{array}{c}61 \\
(0.28)\end{array}$ & $\begin{array}{c}203 \\
(0.47)\end{array}$ & $\begin{array}{c}227 \\
(0.53)\end{array}$ & $p=0.345$ \\
\hline & Case mothers & $\begin{array}{c}19 \\
(0.25)\end{array}$ & $41(0.54)$ & $16(0.21)$ & $\begin{array}{c}79 \\
(0.52)\end{array}$ & $\begin{array}{c}73 \\
(0.48)\end{array}$ & \\
\hline & & AA & $A C$ & CC & A & C & A vs. C \\
\hline \multirow{2}{*}{$\begin{array}{l}\text { CHKA } \\
\quad \text { rs7928739 }\end{array}$} & Control mothers & $\begin{array}{c}72 \\
(0.33)\end{array}$ & $\begin{array}{c}100 \\
(0.47)\end{array}$ & $\begin{array}{c}43 \\
(0.20)\end{array}$ & $\begin{array}{c}244 \\
(0.57)\end{array}$ & $\begin{array}{c}186 \\
(0.43)\end{array}$ & $p=0.924$ \\
\hline & Case mothers & $\begin{array}{c}26 \\
(0.34)\end{array}$ & $35(0.46)$ & $15(0.20)$ & $\begin{array}{c}87 \\
(0.57)\end{array}$ & $\begin{array}{c}65 \\
(0.43)\end{array}$ & \\
\hline \multirow{3}{*}{$\begin{array}{l}\text { CHDH } \\
\text { rs893363 }\end{array}$} & & TT & TC & CC & $\mathbf{T}$ & C & T vs. C \\
\hline & Control mothers & $\begin{array}{c}88 \\
(0.41)\end{array}$ & $\begin{array}{c}97 \\
(0.45)\end{array}$ & $\begin{array}{c}30 \\
(0.14)\end{array}$ & $\begin{array}{l}273 \\
(0.63)\end{array}$ & $\begin{array}{c}157 \\
(0.37)\end{array}$ & $p=0.558$ \\
\hline & Case mothers & $\begin{array}{c}27 \\
(0.36)\end{array}$ & $38(0.50)$ & $11(0.14)$ & $\begin{array}{c}92 \\
(0.60)\end{array}$ & $\begin{array}{c}60 \\
(0.40)\end{array}$ & \\
\hline \multirow{3}{*}{$\begin{array}{l}\text { CHDH } \\
\quad \text { rs2289205 }\end{array}$} & & GG & GA & AA & G & A & G vs. A \\
\hline & Control mothers & $\begin{array}{c}116 \\
(0.54)\end{array}$ & $\begin{array}{c}86 \\
(0.40)\end{array}$ & $\begin{array}{c}13 \\
(0.06)\end{array}$ & $\begin{array}{c}318 \\
(0.74)\end{array}$ & $\begin{array}{c}112 \\
(0.26)\end{array}$ & $p=1.00$ \\
\hline & Case mothers & $\begin{array}{c}43 \\
(0.57)\end{array}$ & $26(0.34)$ & 7 (0.09) & $\begin{array}{c}112 \\
(0.74)\end{array}$ & $\begin{array}{c}40 \\
(0.26)\end{array}$ & \\
\hline
\end{tabular}

aFisher exact test

Table 3. The tests for association* of CHDH, PCYT1A and CHKA polymorphisms in mothers with IUFD incidence and control mothers

\begin{tabular}{|c|c|c|c|c|}
\hline Gene polymorphism & \multicolumn{4}{|c|}{ Risk allele C } \\
\hline & {$[T]$ vs. [C] } & {$[$ [TT] vs. [TC] } & [TT] vs. [CC] & [TT] vs. [TC+ CC] \\
\hline CHDH & $\mathrm{OR}=1.134$ & $\mathrm{OR}=1.277$ & $\mathrm{OR}=1.195$ & $\mathrm{OR}=1.258$ \\
rs893363 & $\mathrm{Cl}=[0.776-1.658]$ & $\mathrm{Cl}=[0.721-2.261]$ & $\mathrm{Cl}=[0.529-2.698]$ & $\mathrm{Cl}=[0.731-2.164]$ \\
& $X^{2}=0.42$ & $X^{2}=0.70$ & $X^{2}=0.18$ & $X^{2}=0.69$ \\
& $\mathrm{p}=0.51625$ & $\mathrm{p}=0.40148$ & $\mathrm{p}=0.66779$ & $\mathrm{p}=0.40751$ \\
\hline
\end{tabular}


Table 3 (cont.). The tests for association* of CHDH, PCYT1A and CHKA polymorphisms in mothers with IUFD incidence and control mothers

\section{Gene polymorphism}

\begin{tabular}{|c|c|c|c|c|}
\hline \multicolumn{5}{|c|}{ Risk allele T } \\
\hline & [C] vs. [T] & {$[\mathrm{CC}]$ vs. [TC] } & [CC] vs. [TT] & {$[\mathrm{TT}+\mathrm{TC}]$ vs. [CC] } \\
\hline $\begin{array}{l}\text { CHDH } \\
\quad \text { rs893363 }\end{array}$ & $\begin{array}{c}\mathrm{OR}=0.882 \\
\mathrm{Cl}=[0.603-1.289] \\
\mathrm{X}^{2}=0.42 \\
\mathrm{p}=0.51625\end{array}$ & $\begin{array}{c}\mathrm{OR}=1.068 \\
\mathrm{Cl}=[0.487-2.345] \\
\mathrm{X}^{2}=0.03 \\
\mathrm{p}=0.86893\end{array}$ & $\begin{array}{c}\mathrm{OR}=0.837 \\
\mathrm{Cl}=[0.371-1.889] \\
\mathrm{X}^{2}=0.18 \\
\mathrm{p}=0.66779\end{array}$ & $\begin{array}{c}\mathrm{OR}=0.958 \\
\mathrm{Cl}=[0.454-2.021] \\
\mathrm{X}^{2}=0.01 \\
\mathrm{p}=0.91079\end{array}$ \\
\hline \multicolumn{5}{|c|}{ Risk allele $\mathrm{A}$} \\
\hline \multirow[b]{2}{*}{$\begin{array}{l}\text { CHDH } \\
\quad \text { rs2289205 }\end{array}$} & [G] vs. [A] & [GG] vs. [GA] & [GG] vs. [AA] & [GG] vs. [GA + AA] \\
\hline & $\begin{array}{c}\mathrm{OR}=1.014 \\
\mathrm{Cl}=[0.666-1.544] \\
\mathrm{X}^{2}=0.00 \\
p=0.94820\end{array}$ & $\begin{array}{c}\mathrm{OR}=1.150 \\
\mathrm{Cl}=[0.649-2.036] \\
\mathrm{X}^{2}=0.23 \\
\mathrm{p}=0.63258\end{array}$ & $\begin{array}{c}\mathrm{OR}=1.453 \\
\mathrm{Cl}=[0.543-3.883] \\
\mathrm{X}^{2}=0.56 \\
\mathrm{p}=0.45482\end{array}$ & $\begin{array}{c}\mathrm{OR}=0.899 \\
\mathrm{Cl}=[0.531-1.523] \\
\mathrm{x}^{2}=0.16 \\
\mathrm{p}=0.69271\end{array}$ \\
\hline \multicolumn{5}{|c|}{ Risk allele G } \\
\hline \multirow[b]{2}{*}{$\begin{array}{l}\text { CHDH } \\
\text { rs2289205 }\end{array}$} & [A] vs. [G] & [AA] vs. [GA] & [AA] vs. [GG] & {$[\mathrm{GG}+\mathrm{GA}]$ vs. $[\mathrm{AA}]$} \\
\hline & $\begin{array}{c}\mathrm{OR}=0.986 \\
\mathrm{Cl}=[0.648-1.501] \\
\mathrm{X}^{2}=0.00 \\
\mathrm{p}=0.94820\end{array}$ & $\begin{array}{c}\mathrm{OR}=0.561 \\
\mathrm{Cl}=[0.203-1.554] \\
\mathrm{X}^{2}=1.26 \\
\mathrm{p}=0.26219\end{array}$ & $\begin{array}{c}\mathrm{OR}=0.688 \\
\mathrm{Cl}=[0.258-1.840] \\
\mathrm{X}^{2}=0.56 \\
\mathrm{p}=0.45482\end{array}$ & $\begin{array}{c}\mathrm{OR}=0.634 \\
\mathrm{Cl}=[0.243-1.655] \\
\mathrm{X}^{2}=0.88 \\
p=0.34868\end{array}$ \\
\hline \multicolumn{5}{|c|}{ Risk allele C } \\
\hline \multirow[b]{2}{*}{$\begin{array}{l}\text { PCYT1A } \\
\text { rs712012 }\end{array}$} & {$[\mathrm{T}]$ vs. $[\mathrm{C}]$} & [TT] vs. [TC] & [TT] vs. [CC] & [TT] vs. $[\mathrm{TC}+\mathrm{CC}]$ \\
\hline & $\begin{array}{c}\mathrm{OR}=0.999 \\
\mathrm{Cl}=[0.684-1.460] \\
\mathrm{x}^{2}=0.00 \\
\mathrm{p}=0.99628\end{array}$ & $\begin{array}{c}\mathrm{OR}=1.150 \\
\mathrm{Cl}=[0.649-2.036] \\
\mathrm{X}^{2}=0.23 \\
\mathrm{p}=0.63258\end{array}$ & $\begin{array}{c}\mathrm{OR}=0.900 \\
\mathrm{Cl}=[0.378-2.142] \\
\mathrm{X}^{2}=0.06 \\
\mathrm{p}=0.81171\end{array}$ & $\begin{array}{c}\mathrm{OR}=1.095 \\
\mathrm{Cl}=[0.632-1.897] \\
\mathrm{X}^{2}=0.10 \\
\mathrm{p}=0.74636\end{array}$ \\
\hline \multicolumn{5}{|c|}{ Risk allele T } \\
\hline \multirow[b]{2}{*}{$\begin{array}{l}\text { PCYT1A } \\
\text { rs712012 }\end{array}$} & {$[\mathrm{C}]$ vs. [T] } & [CC] vs. [TC] & [CC] vs. [TT] & {$[\mathrm{TT}+\mathrm{TC}]$ vs. [CC] } \\
\hline & $\begin{array}{c}\mathrm{OR}=1.001 \\
\mathrm{Cl}=[0.685-1.463] \\
\mathrm{X}^{2}=0.00 \\
\mathrm{p}=0.99628\end{array}$ & $\begin{array}{c}\mathrm{OR}=1.277 \\
\mathrm{Cl}=[0.558-2.921] \\
\mathrm{X}^{2}=0.34 \\
\mathrm{p}=0.56147\end{array}$ & $\begin{array}{c}\mathrm{OR}=1.111 \\
\mathrm{Cl}=[0.467-2.644] \\
\mathrm{X}^{2}=0.06 \\
\mathrm{p}=0.81171\end{array}$ & $\begin{array}{c}\mathrm{OR}=1.207 \\
\mathrm{Cl}=[0.545-2.675] \\
\mathrm{X}^{2}=0.22 \\
\mathrm{p}=0.64235\end{array}$ \\
\hline \multicolumn{5}{|c|}{ Risk allele G } \\
\hline \multirow[b]{2}{*}{$\begin{array}{l}\text { PCYT1A } \\
\text { rs7639752 }\end{array}$} & [A] vs. [G] & [AA] vs. [AG] & [AA] vs. [GG] & {$[A A]$ vs. $[A G+G G]$} \\
\hline & $\begin{array}{c}\mathrm{OR}=0.826 \\
\mathrm{Cl}=[0.571-1.197] \\
\mathrm{X}^{2}=1.02 \\
\mathrm{p}=0.31236\end{array}$ & $\begin{array}{c}\mathrm{OR}=1.007 \\
\mathrm{Cl}=[0.530-1.912] \\
\mathrm{X}^{2}=0.00 \\
\mathrm{p}=0.98294\end{array}$ & $\begin{array}{c}\mathrm{OR}=0.676 \\
\mathrm{Cl}=[0.315-1.452] \\
\mathrm{X}^{2}=1.01 \\
\mathrm{p}=0.31454\end{array}$ & $\begin{array}{c}\mathrm{OR}=0.886 \\
\mathrm{Cl}=[0.482-1.628] \\
\mathrm{X}^{2}=0.15 \\
\mathrm{p}=0.69563\end{array}$ \\
\hline \multicolumn{5}{|c|}{ Risk allele A } \\
\hline \multirow[b]{2}{*}{$\begin{array}{l}\text { PCYT1A } \\
\text { rs7639752 }\end{array}$} & [G] vs. [A] & [GG] vs. [AG] & [GG] vs. [AA] & {$[\mathrm{AA}+\mathrm{AG}]$ vs. [GG] } \\
\hline & $\begin{array}{c}\mathrm{OR}=1.210 \\
\mathrm{Cl}=[0.836-1.752] \\
\mathrm{X}^{2}=1.02 \\
\mathrm{p}=0.31236\end{array}$ & $\begin{array}{c}\mathrm{OR}=1.489 \\
\mathrm{Cl}=[0.771-2.875] \\
\mathrm{X}^{2}=1.41 \\
\mathrm{p}=0.23455\end{array}$ & $\begin{array}{c}\mathrm{OR}=1.478 \\
\mathrm{Cl}=[0.689-3.174] \\
\mathrm{X}^{2}=1.01 \\
\mathrm{p}=0.31454\end{array}$ & $\begin{array}{c}\mathrm{OR}=1.485 \\
\mathrm{Cl}=[0.794-2.778] \\
\mathrm{X}^{2}=1.55 \\
\mathrm{p}=0.21373\end{array}$ \\
\hline \multicolumn{5}{|c|}{ Risk allele C } \\
\hline \multirow[b]{2}{*}{$\begin{array}{l}\text { CHKA } \\
\quad \text { rs7928739 }\end{array}$} & {$[A]$ vs. $[C]$} & [AA] vs. [AC] & [AA] vs. [CC] & {$[A A]$ vs. $[A C+C C]$} \\
\hline & $\begin{array}{c}\mathrm{OR}=0.980 \\
\mathrm{Cl}=[0.674-1.424] \\
\mathrm{X}^{2}=0.01 \\
\mathrm{p}=0.91604\end{array}$ & $\begin{array}{c}\mathrm{OR}=0.969 \\
\mathrm{Cl}=[0.537-1.750] \\
\mathrm{X}^{2}=0.01 \\
\mathrm{p}=0.91745\end{array}$ & $\begin{array}{c}\mathrm{OR}=0.966 \\
\mathrm{Cl}=[0.461-2.023] \\
\mathrm{X}^{2}=0.01 \\
\mathrm{p}=0.92695\end{array}$ & $\begin{array}{c}\mathrm{OR}=0.968 \\
\mathrm{Cl}=[0.557-1.682] \\
\mathrm{X}^{2}=0.01 \\
\mathrm{p}=0.90884\end{array}$ \\
\hline \multicolumn{5}{|c|}{ Risk allele A } \\
\hline \multirow[b]{2}{*}{$\begin{array}{l}\text { CHKA } \\
\text { rs7928739 }\end{array}$} & {$[C]$ vs. $[A]$} & {$[\mathrm{CC}]$ vs. [AC] } & {$[C C]$ vs. [AA] } & {$[A A+A C]$ vs. $[C C]$} \\
\hline & $\begin{array}{c}\mathrm{OR}=1.020 \\
\mathrm{Cl}=[0.702-1.483] \\
\mathrm{X}^{2}=0.01 \\
\mathrm{p}=0.91604\end{array}$ & $\begin{array}{c}\mathrm{OR}=1.003 \\
\mathrm{Cl}=[0.497-2.026] \\
\mathrm{X}^{2}=0.00 \\
\mathrm{p}=0.99259\end{array}$ & $\begin{array}{c}\mathrm{OR}=1.035 \\
\mathrm{Cl}=[0.494-2.168] \\
\mathrm{X}^{2}=0.01 \\
\mathrm{p}=0.92695\end{array}$ & $\begin{array}{c}\mathrm{OR}=1.017 \\
\mathrm{Cl}=[0.527-1.960] \\
\mathrm{x}^{2}=0.00 \\
\mathrm{p}=0.96063\end{array}$ \\
\hline
\end{tabular}

*The tests for association were adapted from http://ihg.gsf.de/cgi-bin/hw/hwa1.pl

$\mathrm{Cl}-95 \%$ confidence interval; OR - odds ratio 
pathology seems to be implicated, such as intrauterine growth retardation (IUGR), preeclampsia, placental abruption and IUFD [21]. Therefore it seems highly advisable to administer folic acid during the second and the third trimester of pregnancy in order to prevent hyperhomocysteinemia which in many cases could be responsible for the vascular damage [22]. Lower folate intake has been linked to reduced cell division, disrupted methylation reactions, and increased inflammatory cytokine production, oxidative stress levels, and apoptosis, all of which could subsequently affect the developing embryo [22]. Thus, the vascular effects related to folate deficiency might also increase the risk of spontaneous abortion and stillbirth. Another explanation is that low folate levels increase the incidence of neural tube defects (NTDs), and fetuses affected with neural tube defects are more commonly aborted spontaneously [23]. While plausible, NTDs are rare conditions and this could only explain a fraction of the association between low folate levels and spontaneous abortion. However, there are other nutritional factors that modify the NTD risk. Periconceptional diet rich in choline reduces NTD occurrence independently of folate intake [24].

As far as we now, this is the first study on correlation between maternal CHKA, PCYT1 A and CHDH gene polymorphisms an the risk of intrauterine fetal death in the Polish population. Beside the basic aim of the study we wanted to highlight nowadays great meaning of nutrigenomics. Nutrigenomics or nutrigenetics, which concerns correlation between genes and diet is a great challenge of worldwide public health. It is very rapidly developing field of science which may influence changing science into practice in human nutrition. Thanks to exploring correlation between genetic changes in metabolic pathways clinicians may be able to provide personalized nutrition recommendations. This is extremely important in case of pregnant women.

Genetically conditioned changes in folate cycle influences the proper course of pregnancy. Because choline metabolism and folate metabolism crosses at the methylation of homocysteine, manipulations that limit folate availability also increase the use of choline in methylation processes. Genetic differences in requirements for choline are due to single nucleotide polymorphisms (SNPs) of choline pathway [25]. But it is worth remarking that polymorphisms of genes involved in folate cycle also modifies the susceptibility to choline deficiency. SNPs in few genes encoding enzymes of folate and choline cycle influence choline metabolism and synthesis. It may result in increased choline dietary demand, especially during pregnancy and lactation. Choline deficiency may alter gene expression through the epigenetics mechanisms. This is another premise of the great meaning of nutrigenomics for worldwide public health. Correlation between nutrition and epigenetics has been already established [26, 27].
That is why we aimed to investigate polymorphisms of choline metabolism pathway in the group of women with a positive history of intrauterine fetal death. Despite the lack of correlation between analyzed in our research genetic variants and the incidence of IUFD we are deeply convinced about the need of further analysis of that issue. Thanks to that it might be possible to identify the subsets of population which differs in a requirement of a specific nutrient [28]. Nutrigenetic profiling will allow to identify subgroups which differs in nutrient requirements and clarify interventions and recommendations. In a consequence, diet guidelines often suggesting unattainable intake of some food will no longer be necessary.

It is also worth to remark some study limitations. We are aware of the need of measuring homocysteine concentration in both study and control group and comparing it to polymorphisms. But due to the long study timing some patients withdrew their consent to research (patients with IUFD were enrolled to the study within 2004-2014 year). Additionally, because of the inconstant concentration of homocysteine in the course of pregnancy in such small group of patient obtained results would not be reliable (in our study IUFD occurrence between 22-40 gestational week).

\section{CONCLUSIONS}

There is no correlation between single CHKA rs7928739, PCYT1A rs712012, PCYT1A rs7639752, CHDH rs893363 and $\mathrm{CHDH}$ rs2289205 polymorphisms and the incidence of intrauterine fetal death. However, revealed statistically significant difference between co-existence of CHKA rs7928739 AC/CHDH rs2289205 AA genotypes between study groups suggest the need of further analysis.

\section{REFERENCES}

1. Zeisel SH, Corbin KD, da Costa KA, et al. Choline: an essential nutrient for humans. Nutrition. 2000; 16(7-8): 669-671.

2. Zeisel SH. Epigenetic mechanisms for nutrition determinants of later health outcomes. Am J Clin Nutr. 2009; 89(5): 1488S-1493S, doi: 10.3945/ajcn.2009.27113B, indexed in Pubmed: 19261726.

3. Jackowski S, Fagone P. CTP: Phosphocholine cytidylyltransferase: paving the way from gene to membrane. J Biol Chem. 2005; 280(2): 853-856, doi: 10.1074/jbc.R400031200, indexed in Pubmed: 15536089.

4. Zeisel SH, da Costa KA. Choline: an essential nutrient for public health. Nutr Rev. 2009;67(11):615-623, doi: 10.1111/j.1753-4887.2009.00246.x, indexed in Pubmed: 19906248.

5. da Costa KA, Sanders LM, Fischer LM, et al. Docosahexaenoic acid in plasma phosphatidylcholine may be a potential marker for in vivo phosphatidylethanolamine $\mathrm{N}$-methyltransferase activity in humans. Am J Clin Nutr. 2011; 93(5): 968-974, doi: 10.3945/ajcn.110.011064, indexed in Pubmed: 21411618.

6. Yan J, Jiang X, West AA, et al. Pregnancy alters choline dynamics: results of a randomized trial using stable isotope methodology in pregnant and nonpregnant women. Am J Clin Nutr. 2013; 98(6): 1459-1467, doi: 10.3945/ajcn.113.066092, indexed in Pubmed: 24132975.

7. Ridgway ND. The role of phosphatidylcholine and choline metabolites to cell proliferation and survival. Crit Rev Biochem Mol Biol. 2013; 48(1): 20 -38, doi: 10.3109/10409238.2012.735643, indexed in Pubmed: 23350810.

8. Aoyama C, Ohtani A, Ishidate K. Expression and characterization of the active molecular forms of choline/ethanolamine kinase-alpha and -beta in mouse tissues, including carbon tetrachloride-induced liver. Biochem J. 2002; 363(Pt 3): 777-784, indexed in Pubmed: 11964179. 
9. Lykidis A, Baburina I, Jackowski S. Distribution of CTP:phosphocholine cytidylyltransferase (CCT) isoforms. Identification of a new CCTbeta splice variant. J Biol Chem. 1999; 274(38): 26992-27001, indexed in Pubmed: 10480912.

10. Lawn JE, Yakoob MY, Haws RA, et al. 3.2 million stillbirths: epidemiology and overview of the evidence review. BMC Pregnancy Childbirth. 2009; 9 Suppl 1: S2, doi: 10.1186/1471-2393-9-S1-S2, indexed in Pubmed: 19426465.

11. Di Mario S, Say L, Lincetto O. Risk factors for stillbirth in developing countries: a systematic review of the literature. Sex Transm Dis. 2007; 34(7 Suppl): S11-S21, doi: 10.1097/01.olq.0000258130.07476.e3, indexed in Pubmed: 17592385.

12. Flenady V, Koopmans $L$, Middleton $P$, et al. Major risk factors for stillbirth in high-income countries: a systematic review and meta-analysis. Lancet. 2011; 377(9774): 1331-1340, doi: 10.1016/S0140-6736(10)62233-7, indexed in Pubmed: 21496916.

13. Gardosi J, Madurasinghe V, Williams M, et al. Maternal and fetal risk factors for stillbirth: population based study. BMJ. 2013;346: f108, indexed in Pubmed: 23349424.

14. Korteweg FJ, Gordijn SJ, Timmer A, et al. A placental cause of intra-uterine fetal death depends on the perinatal mortality classification system used. Placenta. 2008; 29(1): 71-80, doi: 10.1016/j.placenta.2007.07.003, indexed in Pubmed: 17963842.

15. Pariente $G$, Shoham-Vardi I, Kessous $R$, et al. Is stillbirth associated with long-term atherosclerotic morbidity? Am J Obstet Gynecol. 2014; 211(4): 416. e1-416.12, doi: 10.1016/j.ajog.2014.07.032, indexed in Pubmed: 25068554.

16. Aune $D$, Saugstad OD, Henriksen $T$, et al. Maternal body mass index and the risk of fetal death, stillbirth, and infant death: a systematic review and meta-analysis. JAMA. 2014; 311(15): 1536-1546, doi: 10.1001/jama.2014.2269, indexed in Pubmed: 24737366.

17. Choudhary D, Gupta D. Epidemiology of Intrauterine Fetal Deaths: A Study In Tertiary Referral Centre In Uttarakhand. IOSR Journal of Dental and Medical Sciences. 2014; 13(3): 03-06, doi: 10.9790/0853-13320306.

18. Huang DY, Usher RH, Kramer MS, et al. Determinants of unexplained antepartum fetal deaths. Obstet Gynecol. 2000; 95(2): 215-221, indexed in Pubmed: 10674582
19. Yan J, Jiang X, West $A A$, et al. Maternal choline intake modulates maternal and fetal biomarkers of choline metabolism in humans. Am J Clin Nutr. 2012; 95(5): 1060-1071, doi: 10.3945/ajcn.111.022772, indexed in Pubmed: 22418088.

20. Homan GF, Davies M, Norman R. The impact of lifestyle factors on reproductive performance in the general population and those undergoing infertility treatment: a review. Hum Reprod Update. 2007; 13(3): 209-223, doi: 10.1093/humupd/dml056, indexed in Pubmed: 17208948.

21. Daly S, Cotter A, Molloy AE, et al. Homocysteine and folic acid: implications for pregnancy. Semin Vasc Med. 2005; 5(2): 190-200, doi: 10.1055/s-2005-872404, indexed in Pubmed: 16047271.

22. Forges T, Monnier-Barbarino P, Alberto JM, et al. Impact of folate and homocysteine metabolism on human reproductive health. Hum Reprod Update. 2007; 13(3): 225-238, doi: 10.1093/humupd/dml063, indexed in Pubmed: 17307774.

23. Khoshnood $B$, Loane $M$, de Walle $H$, et al. Long term trends in prevalence of neural tube defects in Europe: population based study. BMJ. 2015; 351: h5949, indexed in Pubmed: 26601850.

24. Shaw GM, Carmichael SL, Laurent C, et al. Periconceptional dietary intake of choline and betaine and neural tube defects in offspring. Am J Epidemiol. 2004; 160(2): 102-109, doi: 10.1093/aje/kwh187, indexed in Pubmed: 15234930

25. da Costa KA, Kozyreva OG, Song J, et al. Common genetic polymorphisms affect the human requirement for the nutrient choline. FASEB J. 2006; 20(9): 1336-1344, doi: 10.1096/fj.06-5734com, indexed in Pubmed: 16816108.

26. Feil R. Environmental and nutritional effects on the epigenetic regulation of genes. Mutat Res. 2006; 600(1-2): 46-57, doi: 10.1016/j. mrfmmm.2006.05.029, indexed in Pubmed: 16854438.

27. Drews K. [Folate metabolism--epigenetic role of choline and vitamin B12 during pregnancy]. Ginekol Pol. 2015; 86(12): 940-946, indexed in Pubmed: 26995945.

28. Corbin $\mathrm{KD}$, Zeisel SH. The nutrigenetics and nutrigenomics of the dietary requirement for choline. Prog Mol Biol Transl Sci. 2012; 108: 159-177, doi: 10.1016/B978-0-12-398397-8.00007-1. 